\title{
Cooperation in an asymmetric volunteer's dilemma game with relatedness
}

\author{
HE JunZhou ${ }^{1,2 \dagger}$, WANG RuiWu ${ }^{2 \dagger^{*}}$, CHRISTOPHER X J Jensen ${ }^{3}$, LI YaoTang $^{1 *}$ \& LI ChaoQian ${ }^{1}$ \\ ${ }^{1}$ School of Mathematics and Statistics, Yunnan University, Kunming 650091, China; \\ ${ }^{2}$ State Key Laboratory of Genetic Resources and Evolution, Kunming Institute of Zoology, Chinese Academy of Sciences, Kunming 650223, China; \\ ${ }^{3}$ Department of Mathematics and Science, School of Liberal Arts and Sciences, Pratt Institute, 200 Willoughby Avenue, Brooklyn, NY 11205, USA
}

Received March 16, 2012; accepted March 23, 2012

\begin{abstract}
What motivates some members of a social group to voluntarily incur costs in order to provide for the common good? This question lies at the heart of theoretical and empirical studies of cooperative behavior. This is also the question that underlies the classic volunteer's dilemma model, which has been previously explored in scenarios where group members are related or interact asymmetrically. Here we present a model that combines asymmetry and relatedness, showing that the probability of volunteerism in such systems depends closely on both the degree of asymmetry and level of relatedness between interacting individuals. As has been shown in previous volunteer's dilemma models, the payoff ratio and overall group size also influence the probability of volunteerism. The probability of volunteerism decreases with increasing group size or decreasing cost-to-benefit ratio of the coplayers; in the presence of asymmetrical interactions, subordinate players were more likely to offer public goods than the dominant player. More asymmetrical interactions decrease the probability of volunteerism of the dominant player; overall volunteerism increases with increasing relatedness.
\end{abstract}

asymmetric interaction, cooperation, volunteer's dilemma, relatedness, public goods, kin selection, inclusive fitness

Citation: He J Z, Wang R W, Christopher X J J, et al. Cooperation in an asymmetric volunteer's dilemma game with relatedness. Chin Sci Bull, 2012, 57: 1972-1981, doi: 10.1007/s11434-012-5178-z

For decades, how and why public goods are provided has been a focal question for researchers exploring the evolution of cooperation in animal and microbial social systems (including in human societies) [1-5]. The iterated prisoner's dilemma (IPD) and volunteer's dilemma (VoD) are two of the most influential models used to explain why social partners incur costs to provide common goods shared by all members of the society [6-8]. In the IPD model, individuals are predicted to pay the cost to provide for the common good in an infinitely iterated game, because any defection will discourage further cooperation by other individuals in the future. In the IPD, the predicted optimal behavior for every individual is to provide common public goods, but these predictions fail to explain why common goods are

$\dagger$ These authors contributed equally to this work.

*Corresponding authors (email: ruiwukiz@ hotmail.com; liyaotang@ynu.edu.cn) usually provided by some but not all individual in a social group [9-11].

The volunteer's dilemma (VoD) model, as first proposed in the social sciences [12], is an $N$-person game in which a public good $U$ is produced if and only if at least one player volunteers to pay a cost $K$ (where $U>K>0$ ); when no individual produces the common good, the players involved receive a lower payoff $(0)$ than the payoff $(U-K)$ they would have received if they had volunteered to provide the public good. Obviously, there are $N$ asymmetric equilibria in pure strategies, which are usually not attainable without coordination amongst social partners. In a symmetric mixed Nashequilibrium, the probability of defection is $\beta^{*}=\sqrt[N-1]{K / U}$ [12]. This model assumes that some individuals within the system provide the common goods but every individual will have the same probability $p^{*}=1-\sqrt[N-1]{K / U}$ to be a volunteer 
[12]. However, the production of common goods by the contribution of a single volunteer is inefficient without a mechanism for coordinating who volunteers, which leads to a paradox referred to as the "volunteer's dilemma" $[7,12]$. The probability of free-riding will increase with the group-size $N[12,13]$, and the spread of free-riding has the potential to disrupt the cooperative interaction between the social partners.

The conventional forms of both the IPD and the VoD make the basic assumption that the partners involved in the system interact symmetrically. Under these conditions, all individuals are predicted to have the same probability of paying the cost to provide common goods and maintain the cooperative interaction [14]. However, almost all of the well-studied systems in which cooperative behavior is observed have shown that individuals interact asymmetrically. For example, in the well-known eusocial system of ants and bees, workers provide public services such as nursing and food collection, but queens do not. In the case of inter-specific cooperation, such as the mutualistic relationship between yuccas and the yucca moth and between figs and the fig wasp, the host plant pays the extra cost to maintain the stability of the mutualism through the sanction of non-cooperative pollinators or repression of over-population of pollinators, which prevents the over-exploitation of the common resource shared by pollinators [15-17].

In addition, individuals are often genetically or reciprocally related, which has the potential to affect cooperation $[5,7,10,18]$. In many well-documented systems involving cooperation, the higher the genetic similarity or level of reciprocal exchange (i.e. relatedness), the higher the probability will be for individuals taking a cooperation strategy. For example, in social insects, such bees, ants or wasps, the higher genetically related between the queen and workers, the higher probability of cooperation behaviors could be observed [10].

Other researchers have explored the effects of separately adding either asymmetric interaction or relatedness to the VoD: Diekmann [19] has analyzed asymmetric interaction, and Archetti $[7,18]$ has analyzed relatedness. While these individual treatments of asymmetry and relatedness provided valuable insights, they do not allow us to make predictions about the many systems in which both of these factors simultaneously play a role $[20,21]$. In this paper we modify the classic VoD model to consider the effects of both asymmetry and relatedness, expanding upon and unifying the work of Diekmann [19] and Archetti [18].

\section{Game theoretical analysis}

\subsection{Review of Diekmann's asymmetric volunteer's game}

A provision point version of the classic public goods game, the volunteer's dilemma (VoD) specifies a threshold level of volunteer contribution above which all $N$ players enjoy the benefit of public goods. Each individual in the game maintains two options: (1) to pay the cost of volunteering and thus ensure the provision of public goods to all; or (2) to pay no cost and hope to reap the benefits that come from the volunteerism of others $[12,14,22]$. Such games assume that all individuals pay the same cost for volunteering and reap identical benefits once the public good has been provided. From a biological standpoint this is unrealistic, because most natural interactions are asymmetric, suggesting that not all players earn the same payoff. This asymmetrical interaction can be represented mathematically as different potential net payoffs for different players [16,23-26]. An asymmetric volunteer's dilemma game developed by Diekmann [19] introduces an unequal distribution of costs $K_{i}$ and interests $U_{i}$ among $i=1,2, \cdots, N$ players, and analyzes the binary-decision $N$-person matrix game with each player $i$ 's $(i=1,2, \cdots, N)$ decision an alternative between $C_{i}$ (volunteering, thus cooperation) and $D_{i}$ ( free-riding, thus defection). Assuming that for all $i$ 's $U_{i}>K_{i}>0$, the payoff structure is as follows:

(i) Employing strategy $C_{i}$ always yields the net payoff of $U_{i}-K_{i}$; whereas

(ii) Employing strategy $D_{i}$ yields the maximum payoff of $U_{i}$ whenever at least one other player employs strategy $C_{i}$ ("volunteering" for other players); otherwise

(iii) If all players employ strategy $D_{i}$, then all players forfeit the public good and earn nothing (payoff $=0$ ).

Based on this structure, the asymmetric VoD has $N$ efficient and strict equilibria with exactly one "volunteer" and $N-1$ "free-riders". However, the existence of the pure strategy means there will be only one "volunteer" while the other $N-1$ partners adopt the "free-rider" strategy, an outcome that seems unlikely given that the volunteer must pay the extra cost to provide the public goods but ends up earning the lowest payoff. This feature of the pure-strategy equilibrium leads us to focus on an additional equilibrium point in which mixed (probabilistic) strategies may exist [19].

If we let strategy $D_{i}$ be played with probability $\beta_{i}$, then actor $i$ 's expected utility (i.e. fitness or interest in the biological cooperation systems) $E_{i}$ is

$$
E_{i}=\beta_{i} U_{i}\left(1-\prod_{j \neq i} \beta_{j}\right)+\left(1-\beta_{i}\right)\left(U_{i}-K_{i}\right) .
$$

By partially differentiating with respect to $\beta_{i}$ and setting the equation equal to zero [27,28], we get

$$
\frac{K_{i}}{U_{i}}=\prod_{j \neq i}^{N} \beta_{j}, \quad i=1,2, \cdots, N .
$$

The solution of eq. (2) is

$$
\beta_{i}^{*}=\frac{U_{i}}{K_{i}}\left(\prod_{j=1}^{N} \frac{K_{j}}{U_{j}}\right)^{\frac{1}{N-1}} .
$$


Substitution of $\beta_{i}$ in eq. (1) by $\beta_{i}^{*}$ yields the payoff-vector of the mixed equilibrium strategy, and it becomes apparent that the expected value in the equilibrium $E_{i}^{*}$ does not depend on $\beta_{i}$, hence this is a weak mixed Nash-equilibrium if $0<\beta_{i}^{*}<1$ for $i=1,2, \cdots, N$ [19]. If $U_{i}=U$ and $K_{i}=K$, the mixed equilibrium of the symmetric game is $\beta_{i}^{*}=\sqrt[N-1]{K / U}$ [11]. From eq. (3), the Nash-equilibrium strategy implies that player $i$ 's defection probability will increase with decreasing volunteer cost $\left(K_{i}\right)$ or increasing interest in the collective $\operatorname{good}\left(U_{i}\right)[19]$.

We will study the special case of the asymmetric volunteer's game with one "strong" player and $N-1$ "weak" players with equal degree of weakness in the system, which implies that the "strong" player will enjoy either greater interest $U_{i}$ or lower cost $K_{i}$ than "weak" players [19]. In biological terms, the "strong" role implies social dominance and the "weak" role implies social subordination. We let $K_{s}=K_{1}<K_{2}=$ $K_{3}=\cdots=K_{N}=K_{W}$ and $U_{s}=U_{1} \geqslant U_{2}=U_{3}=\cdots=U_{N}=U_{W}, N>2$. Considering the "strong" player might never volunteer to produce the public goods, such as policing eggs (policing eggs can be treated as public goods of social insect) produced by worker bees but never queens in some systems [20,23], we assume that the "strong" player will take "freerider" strategy if its strength is sufficiently strong.

From eq. (3), we have

$$
\beta_{S}^{*}=\beta_{1}^{*}=\frac{U_{1}}{K_{1}}\left(\frac{K_{1}}{U_{1}} \prod_{j=2}^{N} \frac{K_{j}}{U_{j}}\right)^{\frac{1}{N-1}}=\frac{U_{S}}{K_{S}} \frac{K_{W}}{U_{W}}\left(\frac{K_{S}}{U_{S}}\right)^{\frac{1}{N-1}}
$$

and

$$
\beta_{W}^{*}=\beta_{i}^{*}=\frac{U_{i}}{K_{i}}\left(\prod_{j=1}^{N} \frac{K_{j}}{U_{j}}\right)^{\frac{1}{N-1}}=\left(\frac{K_{S}}{U_{S}}\right)^{\frac{1}{N-1}}, \quad N \geqslant i \geqslant 2,
$$

where $\beta_{\mathrm{S}}^{*}$ and $\beta_{\mathrm{W}}^{*}$ are the defection probability of "strong" player and "weak" co-players (respectively), and $\beta_{\mathrm{W}}^{*}=\beta_{i}^{*}$, $2 \leqslant i \leqslant N$. Based on eqs. (4) and (5), we can conclude that the defection probability of "weak" players will depend on both the cost-to-benefit ratio $K_{\mathrm{S}} / U_{\mathrm{S}}$ of the "strong" player and the group size $N$, while the "strong" player's defection probability is influenced by these same two factors as well as the cost-to-benefit ratio $K_{\mathrm{W}} / U_{\mathrm{W}}$ of "weak" players [19].

For convenience, let $U_{\mathrm{S}}=U_{\mathrm{W}}=1$ and $K_{\mathrm{S}}<K_{\mathrm{W}}<1$. Then

$$
\beta_{\mathrm{S}}^{*}=\beta_{1}^{*}=\frac{K_{\mathrm{W}}}{K_{\mathrm{S}}} K_{\mathrm{S}}^{\frac{1}{N-1}}
$$

and

$$
\beta_{\mathrm{W}}^{*}=\beta_{i}^{*}=K_{\mathrm{S}}^{\frac{1}{N-1}}, \quad N \geqslant i \geqslant 2,
$$

where $K_{\mathrm{S}}$ and $K_{\mathrm{W}}$ are the cost of cooperation to the "strong" player and all "weak" players, respectively.

\subsection{Novel insights into the asymmetric volunteer's game}

Diekmann's model demonstrates that the probability of defection will increase with group size when interaction is asymmetric (Figure 1). However, in this treatment of asymmetry, Diekmann [19] does not analytically explore the effects of varying levels of asymmetry (although he does consider some range of asymmetry in the experimental portion of his paper). We define a new derived parameter, the degree of asymmetry, as $h=K_{\mathrm{W}} / K_{\mathrm{S}}$, and explore below how varying degrees of asymmetry affect the defection probabilities of both "strong" and "weak" players. One issue raised by considering varying degrees of asymmetry (as well as larger group sizes) is that there is a threshold level of asymmetry and group size above which the "strong" player can be predicted to defect with a probability greater than $100 \%$. As can be seen in Figure 1, the predicted probability of defection for the "strong" player can exceed $100 \%$ if group size $(N)$ is sufficiently large in magnitude; an analogous prediction emerges at higher degrees of asymmetry $(h)$. In his analysis of the asymmetrical VoD, Diekmann [19] is careful to specify that his derivations only apply when the defection probability of the "strong" player is less than $100 \%\left(\beta_{\mathrm{S}}^{*}<1\right)$, and never uses parameter values that would produce predicted defection probabilities greater than $100 \%$.

As our goal was to understand the effects of both group size $(N)$ and the degree of asymmetry $(h)$ for all values of these parameters, we sought to determine analytically the threshold value of these parameters above which the

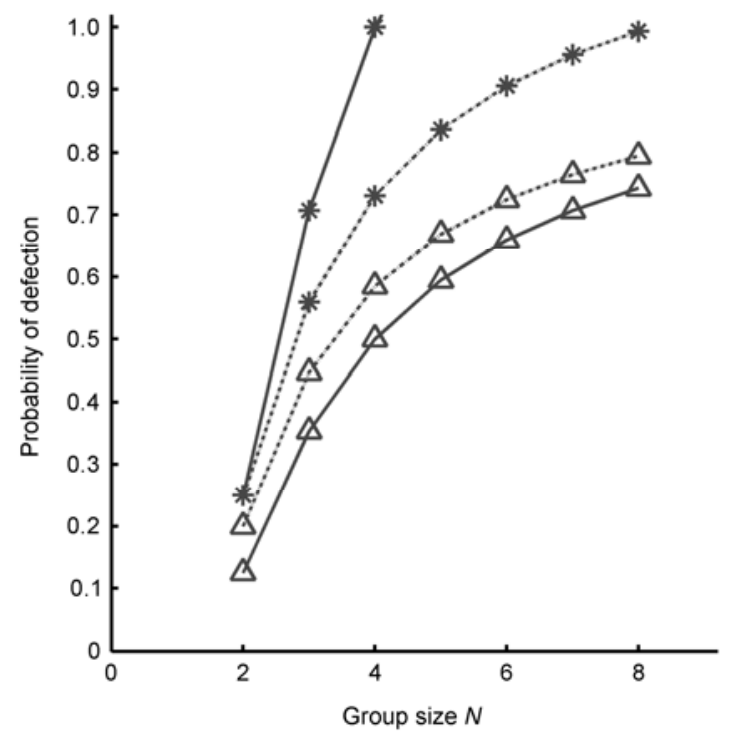

Figure 1 Mixed equilibrium strategy for the asymmetric volunteer's dilemma with one "strong" player (stars) and $N-1$ "weak" players (triangles). If we fix the cost of "weak" players and the "strong" player at $K_{\mathrm{W}}=$ $0.25, K_{\mathrm{S}}=0.2$, then the degree of asymmetry is $h=K_{\mathrm{W}} / K_{\mathrm{S}}=1.25$ (dot lines). If we fix the costs at $K_{\mathrm{W}}=0.25, K_{\mathrm{S}}=0.125$, then the degree of asymmetry is $h=K_{\mathrm{W}} / K_{\mathrm{S}}=2$ (solid lines). For either level of $h$, the defection probabilities of the "strong" player and "weak" co-players as a function of the group-size are $\beta_{\mathrm{S}}^{*}=\beta_{1}^{*}=\left(K_{\mathrm{W}} / K_{\mathrm{S}}\right) K_{\mathrm{S}}^{\frac{1}{N-1}}$ and $\beta_{\mathrm{W}}^{*}=\beta_{i}^{*}=K_{\mathrm{S}}^{\frac{1}{N-1}}$, respectively. 
"strong" player is predicted to defect at a probability greater than $100 \%$. Eq. (6) is greater than one $\left(\beta_{\mathrm{S}}^{*} \geqslant 1\right)$ in the interval $K_{\mathrm{S}}<K_{\mathrm{S}}^{*}=K_{\mathrm{W}}^{(N-1) /(N-2)} \quad\left(\right.$ or $\left.h>h^{*}=K_{\mathrm{W}}^{1 /(2-N)}\right)$. At the critical point $K_{\mathrm{S}}^{*}$, the strength of "strong" player might be sufficiently strong to never volunteers in this situation. Once this critical point $K_{\mathrm{S}}^{*}$ has been reached, the "strong" player will defect with $100 \%$ probability $\left(\beta_{\mathrm{S}}^{*}=1\right)$ for all values of $K_{\mathrm{S}} \leqslant K_{\mathrm{S}}^{*}$, implying that the "strong" player never volunteers. Under this scenario the "strong" player is an invariant freerider, and the asymmetric game collapses to the simpler symmetric volunteer's dilemma with $\mathrm{N}-1$ "weak" players. Accordingly, the defection probability of the "weak" players is $\left(K_{\mathrm{W}}\right)^{\frac{1}{N-2}}$.

Increasing the degree of asymmetry will increase the "strong" player's defection probability but reduce the defection probability of "weak" players in the interval $\left[1, h^{*}\right]$ (Figure 2(b), "stars lines"). From eqs. (6) and (7), we can see that the equilibrium solution for the "strong" player $\beta_{\mathrm{S}}^{*}$ is influenced by the cost of cooperation $K_{\mathrm{S}}$ and the ratio $K_{\mathrm{W}} / K_{\mathrm{S}}$ as well as the group-size $N$; the "weak" players' equilibrium solution $\beta_{\mathrm{W}}^{*}$ is influenced by the cost of cooperation to the "strong" player and also by the group-size. The analysis presented in Figure 2 shows that if the cost to "weak" players $K_{\mathrm{W}}$ and group size $N$ are held constant, the defection probability of "weak" players will increase as the cost of cooperation to the "strong" player $K_{\mathrm{S}}$ increases in interval $\left[K_{\mathrm{S}}^{*}, K_{\mathrm{W}}\right]$ and be fixed at $\left(K_{\mathrm{W}}\right)^{\frac{1}{N-2}}$ in the interval $\left[0, K_{\mathrm{S}}^{*}\right]$ (Figure 2(a), "triangles"); the defection probability of the "strong" player will reach $100 \%$ when the cost of cooperation to the "strong" player is less than the critical point of cost $K_{\mathrm{S}}^{*}$, and it will decrease with increasing cost to the "strong" player when the cost of cooperation to the "strong" player is greater than the critical point of cost $K_{\mathrm{S}}^{*}$ (Figure 2(a), "stars").

Based on our definition of $h$, eqs. (6) and (7) and the above analysis and our assumption for the case in the interval $K_{\mathrm{S}}<K_{\mathrm{S}}^{*}$ (i.e. $h>h^{*}=K_{\mathrm{W}}^{1 /(2-N)}$ ), we can derive the defection probability of the "strong" player and "weak" players as the following functions:

$$
\beta_{\mathrm{S}}^{*}=\left\{\begin{array}{lc}
h\left(\frac{K_{\mathrm{W}}}{h}\right)^{\frac{1}{N-1}}, & \left(1<h<h^{*}=K_{\mathrm{W}}^{1 /(2-N)}\right), \\
1, & \left(h \geqslant h^{*}\right),
\end{array}\right.
$$

and

$$
\beta_{\mathrm{W}}^{*}=\left\{\begin{array}{lc}
\left(\frac{K_{\mathrm{W}}}{h}\right)^{\frac{1}{N-1}}, & \left(1<h<h^{*}=K_{\mathrm{W}}^{1 /(2-N)}\right), \\
\left(K_{\mathrm{W}}\right)^{\frac{1}{N-2}}, & \left(h \geqslant h^{*}\right) .
\end{array}\right.
$$

(a)

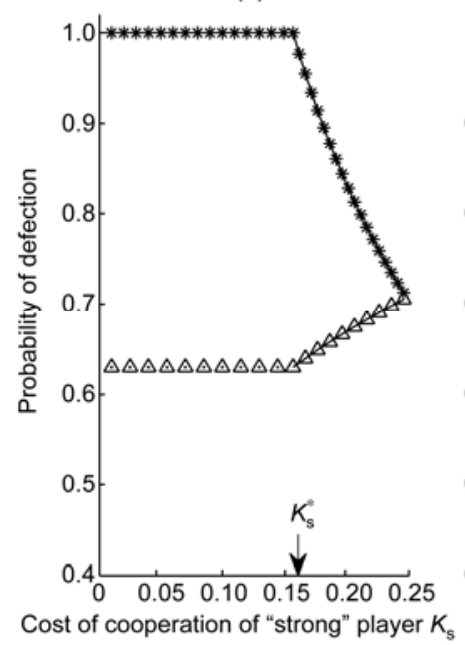

(b)

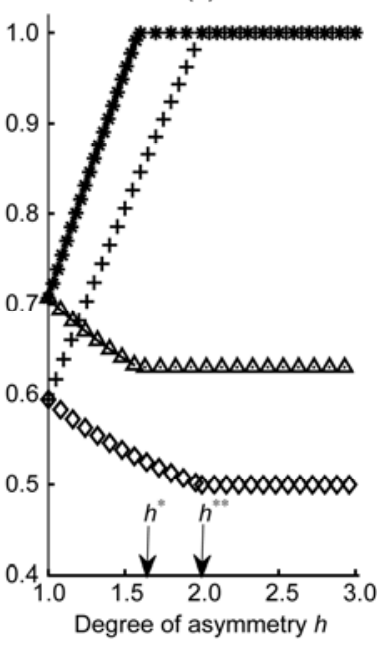

Figure 2 (a) The probability of defection of both "weak" and "strong" players as function of the cost of cooperation for the "strong" player (if we fix the cost to "weak" players at $K_{\mathrm{W}}=0.25$ ). The defection probability of the "strong" player reaches $100 \%$ when the cost to the "strong" player is smaller than the critical point of cost $K_{\mathrm{S}}^{*}$, and it will decrease with increasing cost to the "strong" player; the defection probability of "weak" players increases with increasing cost $K_{S}$ and reach $K_{\mathrm{W}}^{1 /(N-2)}$ when the cost to the "strong" player is smaller than the critical point of cost $K_{\mathrm{S}}^{*}$, where $K_{\mathrm{S}}^{*}=K_{\mathrm{W}}^{(N-1) /(N-2)}$, here at $N=5$. (b) The probability of defection for strong and weak players as functions of the degree of asymmetry when the cost to "weak" players is $K_{\mathrm{W}}=0.25$ and the group-size is $N=5$. The defection probability of the "strong" player will reach $100 \%$ when the degree of asymmetry is greater than $h^{*}$ without relatedness (stars lines) where the critical point $h^{*}=K_{\mathrm{W}}^{1 /(2-N)}$ or greater than $h^{* *}$ with relatedness $r=0.25$ (crosshairs lines) where the critical point $h^{* *}=\left(K_{\mathrm{W}} /[r(N-1)+1]^{1 /(2-N)}\right.$. The defection probability of "weak" players without relatedness (triangles lines) is greater than with relatedness (diamonds lines), and it will decrease with increasing asymmetry when the asymmetry less than $h^{*}\left(\right.$ or $\left.h^{* *}\right)$ and will reach $K_{\mathrm{W}}^{1 /(N-2)}\left(\right.$ or $\left.\left(K_{\mathrm{W}} /(r(N-1)+1)\right)^{1 /(N-2)}\right)$ when the degree of asymmetry greater than $h^{*}$ (or $\left.h^{* *}\right)$.

As these functions make clear, the probability of defection of the "strong" player will increase with increasing asymmetry (Figure 2(b), "stars"), but it will reach $100 \%$ when the degree of asymmetry is greater than the critical point of the degree of asymmetry $h^{*}$, where $h^{*}=K_{\mathrm{W}}^{1 /(2-N)}$. The probability of defection of "weak" players will decrease with increasing degree of asymmetry $h$ in the interval $\left[1, h^{*}\right]$ and be fixed at $\left(K_{\mathrm{W}}\right)^{\frac{1}{N-2}}$ when the degree of asymmetry is greater than the critical point $h^{*}$ (Figure 2(b), "triangles").

\subsection{Asymmetric volunteer's dilemma game with relatedness}

Individuals in an asymmetric system in which cooperation can occur are often genetically or reciprocally related [29]. Relatedness has the potential to increase cooperation within the social group $[5,7,10,18]$. In the VoD game, Archetti $[7,18]$ has also demonstrated that relatedness strongly influences 
the likelihood that players will exhibit cooperative behavior. In fact, both the effects of asymmetry and relatedness may simultaneously coexist in many real systems, such as eusocial system of ants and bees [20], inter-specific mutualistic of the yuccas and the yucca moth [15] and the figs and the fig wasp $[16,17,21,30]$. Here, we sought to explore how asymmetry combined with relatedness affects the behaviors of individuals in cooperative social systems by recombining and extending the models of Diekmann [19] and Archetti [7,18].

In the asymmetric volunteer's dilemma game developed here, we study the special case of the asymmetric volunteer's game with one "strong" player and $N-1$ "weak" players with equal degree of weakness in the system, assuming that the $N$ players are genetically and/or reciprocally related. Payoffs are similar to those shown above (see Section 1.1) for the special case of the asymmetric volunteer's game with one "strong" player and $N-1$ "weak" players with equal degree of weakness. All parameters used below are the same as those used above for the asymmetrical case without relatedness. For convenience, we let $r$ be the average relatedness to other members of the group. The probability that $l$ of the "weak" players fail to volunteer can be expressed from the perspective of the "strong" player in relation to all "weak" players as:

$$
f_{l}=\left(\begin{array}{c}
N-1 \\
l
\end{array}\right) \beta_{\mathrm{W}}^{l}\left(1-\beta_{\mathrm{W}}\right)^{N-1-l} .
$$

The probability that $j$ of the "weak" players fail to volunteer can be expressed from the perspective of a single "weak" player in relation to the remaining $N-2$ "weak" players as:

$$
g_{j}=\left(\begin{array}{c}
N-2 \\
j
\end{array}\right) \beta_{\mathrm{W}}^{j}\left(1-\beta_{\mathrm{W}}\right)^{N-2-j} .
$$

The fitness of the "strong" player who chooses to provide the public good is then

$$
\begin{aligned}
E_{\mathrm{S}}^{V} & =\overbrace{\{\overbrace{U_{\mathrm{S}}-K_{\mathrm{S}}}^{\text {self }}+}^{\underbrace{\beta_{\mathrm{w}}{ }^{N-1}(N-1) U_{\mathrm{w}}}_{\text {nobody }}+\underbrace{\sum_{i=0}^{N-2} f_{l}\left[(N-1-l)\left(U_{\mathrm{w}}-K_{\mathrm{W}}\right)+l U_{\mathrm{w}}\right]}_{\text {volunteers }}\}}\}
\end{aligned}
$$

Here the "strong" volunteer has a minimum direct payoff of $U_{S}-K_{S}$ regardless of whether or not one of the "weak" players produces the public good. In this case, if none of the other $N-1$ members of the group (whose average relatedness to the focal individual is $r$ ) produces the common good (which happens with probability $\beta_{W}^{N-1}$ ) the payoff to "weak" players will be $U_{W}$, and the payoff to the "strong" player will be $U_{S}-K_{S}$. If $l$ of these $N-1$ individuals fail to provide the public good (the probability of which is $f_{i}$ ), the payoff for those $N-1-l$ who volunteer to provide the public good is $U_{W}-K_{W}$ and the payoff for the $l$ who refuse to provide the public good is $U_{W}[7,18]$.

The fitness of the "strong" player who refuses to provide the public good is

$$
\begin{aligned}
E_{S}^{I} & =\overbrace{\underbrace{\left(1-\beta_{W}^{N-1}\right) U_{S}}_{\text {somebody }}+\underbrace{\beta_{W}^{N-1} \cdot 0}_{\text {volunteers }}}^{\text {self }}+ \\
& \overbrace{\{\underbrace{\beta_{W}^{N-1}(N-1) \cdot 0}_{\text {nobody volunteers }}+\underbrace{\sum_{i=0}^{N-2} f_{l}\left[(N-1-l)\left(U_{W}-K_{W}\right)+l U_{W}\right]}_{\text {kin }}\}}^{\text {volunteers }}\}
\end{aligned}
$$

The "strong" player who defects has a direct payoff $U_{S}$ so long as one of the "weak" players produces the public good, which happens with probability $1-\beta_{W}^{N-1}$. The payoff of the "strong" player is 0 if none of the "weak" players produces the public good, which happens with probability $\beta_{W}^{N-1}$. In this case, if none of the other $N-1$ members of the group (whose average relatedness to the focal individual is $r$ ) volunteers to provide the public good (which happens with probability $\beta_{W}^{N-1}$ ), each “weak" player's payoff is 0 . If instead $l$ of these $N-1$ individuals fail to provide the public good (which happens with probability $f_{l}$ ), the payoff for those $N-1-l$ players who produce the public good is $U_{W}-K_{W}$ and the payoff for the $l$ players who do not produce the public good is $U_{W}[7,18]$.

As with the "strong" player, the fitness of individual "weak" players for the two pure strategies of providing and not providing the public good can be written as:

$$
E_{\mathrm{w}}^{V}=\overbrace{U_{\mathrm{w}}-K_{\mathrm{w}}}^{\text {self }}+
$$

$r\{\underbrace{\beta_{\mathrm{S}}^{\beta_{\mathrm{W}}^{N-2}\left[(N-2) U_{\mathrm{w}}+U_{\mathrm{S}}\right]}}_{\text {nobody volunters }}+\underbrace{\sum_{j=0}^{N-3}\left\{\beta_{\mathrm{S}} g_{j}\left[(N-2-j)\left(U_{\mathrm{w}}-K_{\mathrm{W}}\right)+j U_{\mathrm{w}}+U_{\mathrm{S}}\right]\right\}+\sum_{j=0}^{N-2}\left\{\left(1-\beta_{\mathrm{S}}\right) g_{j}\left[(N-2-j)\left(U_{\mathrm{w}}-K_{\mathrm{W}}\right)+j U_{\mathrm{w}}+\left(U_{\mathrm{S}}-K_{\mathrm{S}}\right)\right]\right\}}_{\text {somebody }}\}$ 


$$
\begin{aligned}
& E_{\mathrm{W}}^{I}=\overbrace{\underbrace{\left(1-\beta_{\mathrm{S}} \beta_{\mathrm{w}}{ }^{N-2}\right) U_{\mathrm{w}}}_{\text {somebody }}+\underbrace{\beta_{\mathrm{S}} \beta_{\mathrm{w}}{ }^{N-2} \cdot 0}_{\text {nobunters }}}^{\text {self }}+ \\
& r \overbrace{\{\underbrace{\beta_{\mathrm{S}} \beta_{\mathrm{W}}^{N-2}[(N-2) \cdot 0+0]}_{\text {nobody }}+\underbrace{\sum_{j=0}^{N-3}\left\{\beta_{\mathrm{S}} g_{j}\left[(N-2-j)\left(U_{\mathrm{W}}-K_{\mathrm{W}}\right)+j U_{\mathrm{W}}+U_{\mathrm{S}}\right]\right\}+\sum_{j=0}^{N-2}\left\{\left(1-\beta_{\mathrm{S}}\right) g_{j}\left[(N-2-j)\left(U_{\mathrm{W}}-K_{\mathrm{W}}\right)+j U_{\mathrm{W}}+\left(U_{\mathrm{S}}-K_{\mathrm{S}}\right)\right]\right\}}_{\text {volunters }}\}}^{\text {kin }}\}
\end{aligned}
$$

The mixed-strategy equilibrium can be found by equating the fitness of the two pure strategies of the "strong" player and "weak" players $[7,18]$, which produces the solution

$$
\left\{\begin{array}{l}
\beta_{\mathrm{W}}^{* *}=\left(\frac{K_{\mathrm{S}}}{r(N-1) U_{\mathrm{S}}+U_{\mathrm{S}}}\right)^{\frac{1}{N-1}}, \\
\beta_{\mathrm{S}}^{* *}=\frac{K_{\mathrm{W}}}{r\left[(N-2) U_{\mathrm{W}}+U_{\mathrm{S}}\right]+U_{\mathrm{W}}}\left(\frac{r\left[(N-1) U_{\mathrm{W}}+U_{\mathrm{S}}\right]}{K_{\mathrm{S}}}\right)^{\frac{N-2}{N-1}},
\end{array}\right.
$$

where $\beta_{\mathrm{S}}^{* *}$ and $\beta_{\mathrm{W}}^{* *}$ are the probability of defection of the "strong" player and all "weak" players at equilibrium, respectively.

Equivalently, we have

$$
\left\{\begin{aligned}
\beta_{\mathrm{W}}^{* *}= & \left(\frac{K_{\mathrm{S}}}{r(N-1) U_{\mathrm{W}}+U_{\mathrm{S}}}\right)^{\frac{1}{N-1}}, \\
\beta_{\mathrm{S}}^{* *}= & \frac{K_{\mathrm{W}}}{r\left[(N-2) U_{\mathrm{W}}+U_{\mathrm{S}}\right]+U_{\mathrm{W}}} \frac{r(N-1) U_{\mathrm{W}}+U_{\mathrm{S}}}{K_{\mathrm{S}}} \\
& \left(\frac{K_{\mathrm{S}}}{r(N-1) U_{\mathrm{W}}+U_{\mathrm{S}}}\right)^{\frac{1}{N-1}},
\end{aligned}\right.
$$

where $r(N-1) U_{\mathrm{W}}+U_{\mathrm{S}}$ and $K_{\mathrm{S}}$ can be defined as the benefit and the cost of cooperation (respectively) for the "strong" player if none of the other $N-1$ members of the group produces the public good. If we let $B_{\mathrm{S}}=r(N-1) U_{\mathrm{W}}+U_{\mathrm{S}}$ and $C_{\mathrm{S}}=K_{\mathrm{S}}$, then $\frac{C_{\mathrm{S}}}{B_{\mathrm{S}}}=\frac{K_{\mathrm{S}}}{r(N-1) U_{\mathrm{W}}+U_{\mathrm{S}}}$ is the indirect cost-tobenefit ratio for the cooperating "strong" player (correspondingly, $K_{\mathrm{S}} / U_{\mathrm{S}}$ is the direct cost-to-benefit ratio for the cooperating "strong" player). Using similar notations for the $B_{\mathrm{W}}$ and $C_{\mathrm{W}}$ of each "weak" player, $C_{\mathrm{W}} / B_{\mathrm{W}}=K_{\mathrm{W}} / r\left[(N-2) U_{\mathrm{W}}+\right.$ $\left.U_{\mathrm{S}}\right]+U_{\mathrm{W}}$, yielding the following simplified defection probabilities:

$$
\left\{\begin{array}{l}
\beta_{\mathrm{W}}^{* *}=\left(\frac{C_{\mathrm{S}}}{B_{\mathrm{S}}}\right)^{\frac{1}{N-1}}, \\
\beta_{\mathrm{S}}^{* *}=\frac{C_{\mathrm{W}}}{B_{\mathrm{W}}} \frac{B_{\mathrm{S}}}{C_{\mathrm{S}}}\left(\frac{C_{\mathrm{S}}}{B_{\mathrm{S}}}\right)^{\frac{1}{N-1}} .
\end{array}\right.
$$

With the above notations, it is clear that the indirect costto-benefit ratio will decrease with increasing relatedness within the group. The probability of defection depends on the group-size $N$ and the cost-to-benefit ratio of the other players. If we remove the asymmetry by setting $K_{\mathrm{S}}=K_{\mathrm{W}}$, the results simplify to those of Archetti [7,18]. If we remove relatedness by setting $r=0$, the results simplify to those of Diekmann [19]. These results demonstrate that the model developed here is a general form that subsumes these two more specific variants of the volunteer's dilemma.

For convenience, let $U_{\mathrm{S}}=U_{\mathrm{W}}=1$, and $K_{\mathrm{S}}<K_{\mathrm{W}}<1$. This produces the equilibrium probabilities of defection

$$
\left\{\begin{array}{l}
\beta_{\mathrm{W}}^{* *}=\left(\frac{K_{\mathrm{S}}}{r(N-1)+1}\right)^{\frac{1}{N-1}}, \\
\beta_{\mathrm{S}}^{* *}=\frac{K_{\mathrm{W}}}{K_{\mathrm{S}}}\left(\frac{K_{\mathrm{S}}}{r(N-1)+1}\right)^{\frac{1}{N-1}} .
\end{array}\right.
$$

Using our previous notation letting $h=K_{\mathrm{W}} / K_{\mathrm{S}}$ be the degree of asymmetry, then

$$
\left\{\begin{array}{l}
\beta_{\mathrm{W}}^{* *}=\left(\frac{K_{\mathrm{W}}}{h} \frac{1}{r(N-1)+1}\right)^{\frac{1}{N-1}} \\
\beta_{\mathrm{S}}^{* *}=h\left(\frac{K_{\mathrm{W}}}{h} \frac{1}{r(N-1)+1}\right)^{\frac{1}{N-1}} .
\end{array}\right.
$$

From eq. (14), the probability of defection of the "strong" player will reach $100 \%$ at the point $h^{* *}$ (where $\left.h^{* *}=\left(\frac{K_{\mathrm{W}}}{r(N-1)+1}\right)^{\frac{1}{2-N}}\right)$, and $\beta_{\mathrm{S}}^{* *} \geqslant 1$ in the interval $h \geqslant h^{* *}$ (Figure 2(b), "crosshairs lines"). This discovery mirrors our discovery for eq. (8) as discussed in Section 1.2. When the "strong" player defects with $100 \%$ probability, the game once again collapses to a symmetric game involving $\mathrm{N}-1$ players (who are related in this scenario), and the defection probability of the "weak" players is $\left(\frac{K_{\mathrm{W}}}{r(N-1)+1}\right)^{\frac{1}{N-2}}$ (Figures 2(b) and 3(c)).

The probability of defection at equilibrium will depend closely on the relatedness, the degree of asymmetry between members of the group, and the group-size. The prob- 
ability of defection will increase with increasing group size (Figure 3(a)) [12,19] and decrease with increasing relatedness (Figure 3(b)) for both the "strong" and "weak" players. As the degree of asymmetry increases, the probability of defection of the "strong" player will increase, while "weak" players are less likely to defect when the degree of asymmetry is less than the critical point $h^{* *}$ (Figure 3(c)). When the cost to "weak" players and group size are held constant, the defection probability of the "strong/weak" player reaches $100 \% /\left(K_{\mathrm{w}} / r(N-1)+1\right)^{\frac{1}{N-2}}$ whenever the degree of asymmetry is greater than the critical point $h^{* *}$, and this critical point will increase in value with increasing relatedness (Figure 2(b)). For all parameter ranges, the probability of defection of the "strong" player is always greater than that of the "weak" players [19].

In Figure 4, the flat-roofed areas (in dark red) represent the parameter ranges of non-cooperation for the "strong" player. Increasing the relatedness or decreasing the degree of asymmetry and/or group-size will reduce the areas of non-cooperation for the "strong" player.

\section{Discussion}

A great number of social systems-including many human societies-involve voluntary contributions by individuals to the common good. Identifying what factors determine which individuals will make these contributions remains an active area of both theoretical and empirical inquiry. Previous work has demonstrated the importance of the cost-to-benefit ratio and overall group size $[12,19]$ and genetic or reciprocal relationships $[7,18]$ in determining how volunteer behaviors might evolve. However, these theories assume that the interactions between members of the group are symmetrical, which makes it difficult to account for the uneven contributions often observed in natural systems. Asymmetric contributions to the common good have been observed in humans [9], bees [20], mole-rats [23], and a number of inter-specific mutualisms $[15,17,26,30]$. However, theoretical work has not shed light on how social asymmetries affect predicted patterns of volunteerism when members of the social group are related.

We present here just such an asymmetric model. Our model produces the same results as Diekmann's, showing that the mixed Nash-equilibrium predicts a higher probability of defection for the "strong" player than the "weak" coplayers [19] in absence of relatedness ( $r=0$ in our model). However, our model yields an additional insight: that for the "weak" player the defection probability negatively correlates with the degree of asymmetry, whereas the same correlation is positive for the "strong" player (Figure 2(b)). The mixed-equilibrium strategy yields the optimal payoff, which is higher for "strong" players who incur lower costs. In order to earn the maximum payoff, the "strong" player's defection probability has to be greater than the defection (a) $r=0.25 ; h=1.25$

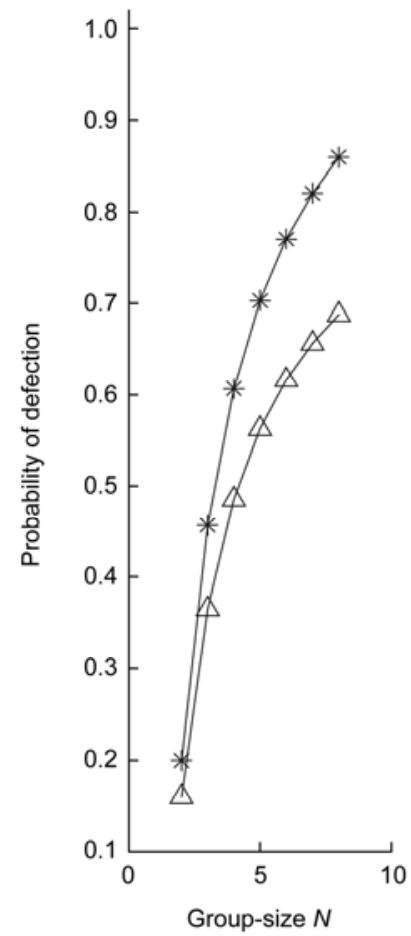

(b) $h=1.25 ; N=5$

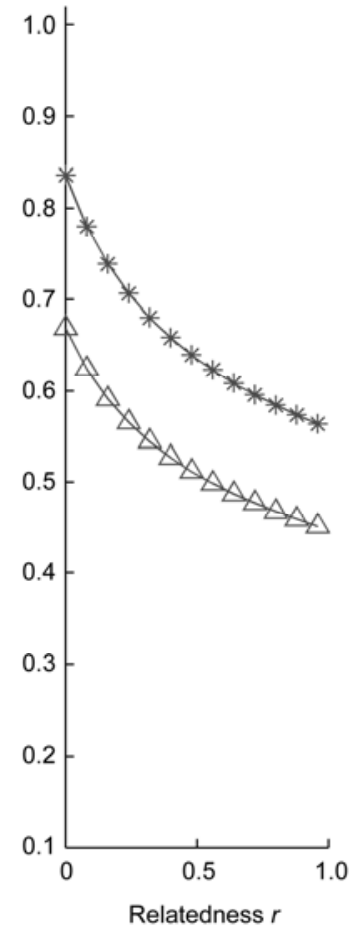

(c) $r=0.25 ; N=5$

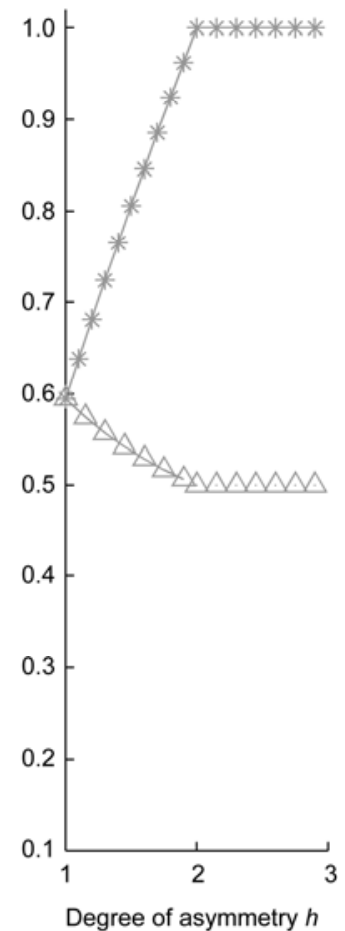

Figure 3 The probability of defection for "strong" (stars) and "weak" (triangles) players as a function of the (a) group-size $N$, (b) the relatedness $r$, and (c) the degree of asymmetry $h$ when other parameters are fixed at $r=0.25, K_{\mathrm{W}}=0.25, h=1.25$ and $N=5$. 


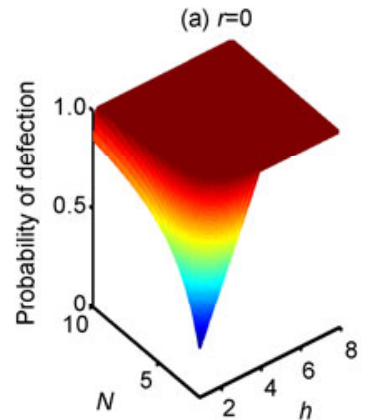

(d) $h=1$

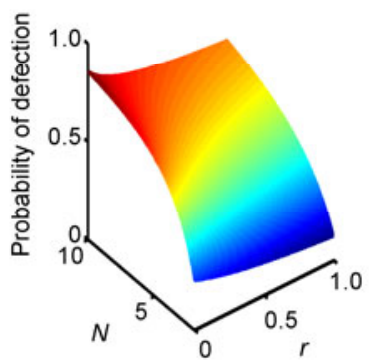

(b) $r=0.25$

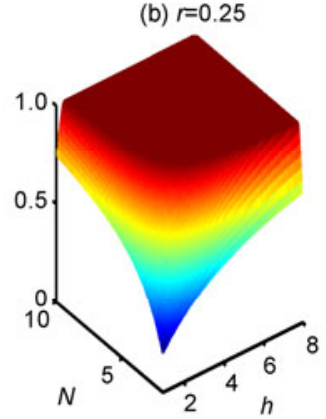

(e) $h=2$

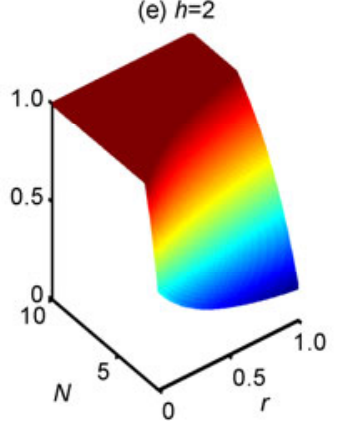

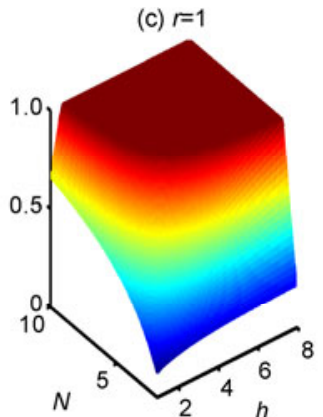

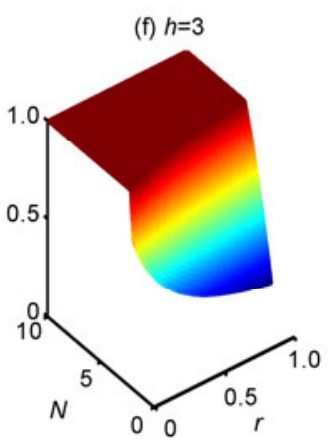

Figure 4 The defection probability of strong player $\alpha^{*}$ as a function of the group-size $N$ and the degree of asymmetry $h$ for different levels of relatedness $r$ $(\mathrm{a}-\mathrm{c})$, and as function of the group-size $N$ and relatedness $r$ for different degrees of asymmetry $h$ (d-f) in an asymmetric volunteer dilemma game, where the cost of cooperating to the "weak" players is fixed at $K_{\mathrm{W}}=0.25$. The color bar represents defection probabilities of the strong player ranging from zero to one.

probability of "weak" co-players, who maintain a lower maximum payoff [19]. This suggests that as the degree of asymmetry increases, the "strong" player may have an increasing incentive to punish non-cooperating "weak" players.

Asymmetric social interactions have the potential to encourage cooperation, which can be achieved through the sanction and/or repression of a subordinate or dependent symbiont by the dominant or host $[8,17,31,32]$. Subordinates will cooperate if the benefits they receive offset the costs they incur due to punishment or sanction by a dominant, allowing the dominant to exploit the subordinates. Higher genetic or reciprocity relatedness, which can be created by limited dispersal ability and/or high expected benefit for the involved partners [1,10], might make sanction and/or repression more profitable for the donor individual [26].

That varying degrees of asymmetry and relatedness will lead to different volunteer strategies among social partners suggests an explanation for why in some species of social insects queens police eggs while in other species workers police eggs [33-37]: opposite policing strategies may result from differences in the degree of asymmetry and relatedness across species. Thus, the degree of asymmetry between dominants and subordinates in Apis florae may approach the critical point $h^{* *}$ (Figure 2(b)), whereas in Apis mellifera the degree of asymmetry may be lower than the critical point $h^{* *}[33,34]$, where $h^{* *}$ is greatly affected by relatedness (see Figure 2(b)). This difference in effective asymmetry may explain fundamental differences in the way policing works in these two species of superorganisms. In our model, symmetric interaction (i.e. degree of asymmetry $h=1$ ) makes voluntary contributions to common goods least likely. This might explain experiments demonstrating that in the ponerine ant (Pachycondyla inverse) policing behavior is reduced when the queen is removed [36]. Our model also shows that the "strong" volunteer has a greater impact when the degree of asymmetry is higher. This result is consistent with empirical observations showing that in some species, queen policing (a behavior that occurs within a highly asymmetric interaction) almost completely prevents workers from reproducing [37-39].

Another critical finding of our model is that defection will be the "strong" player's dominant strategy when the degree of asymmetry approaches the critical point $h^{*}\left(\right.$ or $\left.h^{* *}\right)$, but cooperation will be dominant strategy of the "weak" players. This result is only somewhat tempered if the "strong" and "weak" players are related (Figure 2(b)). This may have led to the evolution of dominant behavior strategies aimed at coercing cooperation from subordinates when the degree of asymmetry is very high. In those insect societies that are headed by a single once-mated queen, the degree of asymmetry might be higher than in those with multiple queens. In the single-queen systems, the degree of asymmetry may approach the critical point, leading queens to use pheromones to manipulate workers [40,41], which leads to more prevalent worker policing in these species [37]. The experimental results of the model developed here may help explain the different strategies of different insect 
societies.

In field observations, "weak" players, the young or subordinate members of the group, tend to offer public goods. For example, in the social groups of meerkats (Suricata suricatta), the alarm calls of young are longer in duration, more modulated, noisier, and have their energy at higher frequencies than those of adults $[42,43]$. These phenomena are consistent with the findings of our model, as weak players are more likely to contribute to public goods. If considering the cost of cooperation is one means of explaining individual interactions [44], then understanding and assessing the degree of asymmetry may be important in explaining the pattern of cooperation observed in many social systems.

Our model shows that relatedness, which could be construed as either genetic or reciprocity relatedness [45], will also facilitate cooperation, even when related individuals interact asymmetrically (Figures 2(b) and 3(b)). However, it is important to point out that relatedness is not the primary factor dictating the probability of volunteering for each player (as shown in Figure 3(b)), but it has the potential to modify this probability. The effect of relatedness upon the "strong" player is greater than on the "weak" player: that is, $\left|\frac{\partial \beta_{\mathrm{S}}{ }^{* *}}{\partial r}\right|>\left|\frac{\partial \beta_{\mathrm{W}}{ }^{* *}}{\partial r}\right|$. Our model is consistent with other $N$-person cooperative models $[7,12,18,19,46,47]$ in that it shows that the probability of defection will increase with increasing group-size for both "strong" and "weak" players (Figures 1 and 3(a)). This general result was predicted by Goeree et al. (from http://citeseerx.ist.psu.edu/viewdoc/download?doi=10. 1.1.77.7999\&rep=rep1\&type=pdf), who argued that the Nash equilibrium predicts the probability of volunteering to be a decreasing function of group-size. Recently, Healy et al. [22] have also provided experimental evidence to support this pattern.

In summary, our model demonstrates that group-size, cost-to-benefit ratio, relatedness, and the degree of asymmetry all influence the probability of volunteerism in a system where individuals can sacrifice for the common good. In our asymmetric volunteer's dilemma game with relatedness, the "strong" and "weak" players employ a mixed strategy of either donation or defection when the group-size and/or the degree of asymmetry are lower than threshold values (Figure 4); the "weak" players have more incentive to produce the public good than the "strong" player, incentivizing the "strong" player to exploit its "weak" co-players because the asymmetric interaction allows the "strong" player to coerce "weak" players into supplying public goods. Relatedness has the overall effect of increasing the level of volunteerism, increasing the overall level of cooperation in the social group.

We are indebted to Carole Sirovich, Zhan-Shan Ma, Ya-Qiang Wang, QiLong Liu, Lei Gao, Qiu-Xia Zhao and Bao-Fa Sun for their discussion and comments during the preparation and revision of this manuscript. This work was supported by the National Natural Science Foundation of China (31170408, 71161020, 10961027), the Program for Innovative Research Team (in Science and Technology) in University of Yunnan Province, the Natural Science Foundation of Yunnan Province (2009CD104), the West Light Foundation of the Chinese Academy of Sciences, the Special Fund for the Excellent Youth of the Chinese Academy of Sciences (KSCX2-EW$Q-9)$, and the State Key Laboratory of Genetic Resources and Evolution.

1 Axelrod R, Hamilton W D. The evolution of cooperation. Science, 1981, 211: 1390-1396

2 Milinski M. Tit for tat in sticklebacks and the evolution of cooperation. Nature, 1987, 325: 433-435

3 Colman A M. Game Theory and Its Applications in the Social and Biological Sciences. Oxford: Butterworth-Heinemann, 1995

4 Dugatkin L A. Cooperation Among Animals: An Evolutionary Perspective. Oxford: Oxford University Press, 1997

5 West S A, Pen I, Griffin A S. Cooperation and competition between relatives. Science, 2002, 296: 72-75

6 Hauert C, Szabó G. Prisoner's dilemma and public goods games in different geometries: Compulsory versus voluntary interactions. Complexity, 2003, 8: 31-38

7 Archetti M. The volunteer's dilemma and the optimal size of a social group. J Theor Biol, 2009, 261: 475-480

8 Nikiforakis N, Normann H T, Wallace B. Asymmetric enforcement of cooperation in a social dilemma. S Econ J, 2010, 76: 638-659

9 Axelrod R. The Evolution of Cooperation. New York: Basic Books, 1984

10 Frank S A. Foundations of Social Evolution Princeton. New Jersey: Princeton University Press, 1998

11 West S A, Griffin A S, Gardner A. Social semantics: Altruism, cooperation, mutualism, strong reciprocity and group selection. J Evol Biol, 2007, 20: 415-432

12 Diekmann A. Volunteer's dilemma. J Confl Resol, 1985, 29: 605610

13 Darley J M, Latane B. Bystander intervention in emergencies: Diffusion of responsibility. J Pers Soc Psychol, 1968, 8: 377-383

14 Otsubo H, Rapoport A. Dynamic volunteer's dilemmas over a finite horizon-An experimental study. J Confl Resol, 2008, 52: 961-984

15 Pellmyr O, Huth C J. Evolutionary stability of mutualism between yuccas and yucca moths. Nature, 1994, 372: 257-260

16 Wang R W, Ridley J, Sun B F, et al. Interference competition and high temperatures reduce the virulence of fig wasps and stabilize a fig-wasp mutualism. PLoS ONE, 2009, 4: e7802

17 Wang R W, Sun B F, Zheng Q. Diffusive co-evolution and mutualism maintenance mechanisms in a fig-fig wasp system. Ecology, 2010, 91: 1308-1316

18 Archetti M. Cooperation as a volunteer's dilemma and the strategy of conflict in public goods games. J Evol Biol, 2009, 22: 2192-2200

19 Diekmann A. Cooperation in an asymmetric volunteer's dilemma game theory and experimental evidence. Int J Game Theory, 1993, 22: 75-85

20 Ratnieks F L W, Wenseleers T. Altruism in insect societies and beyond: Voluntary or enforced? Trends Ecol Evol, 2008, 23: 45-52

21 Wang R W, Sun B F, Zheng Q, et al. Asymmetric interaction and indeterminate fitness correlation between cooperative partners in the fig-fig wasp mutualism. J R Soc Interface, 2011, 8: 1487-1496

22 Healy A, Pate J. Asymmetry and incomplete information in an experimental volunteer's dilemma. 18th World IMACS/MODSIM Congress, Cairns, Australia, 2009. 1457-1462

23 Reeve H K. Queen activation of lazy workers in colonies of the eusocial naked mole-rat. Nature, 1992, 358: 147-149

24 Heinsohn R, Packer C. Complex cooperative strategies in group-territorial African lions. Science, 1995, 269: 1260-1262

25 Wang R W, Shi L. The evolution of cooperation in asymmetric systems. Sci China Life Sci, 2010, 53: 139-149

26 Wang R W, He J Z, Wang Y Q, et al. Asymmetric interaction will facilitate the evolution of cooperation. Sci China Life Sci, 2010, 53: 
1041-1046

27 Harsanyi J C, Slten R. A General Theory of Equilibrium Selection in Games. Cambridge, MA: MIT Press, 1988

28 Binmore K G. Playing Fair: Game Theory and the Social Contract. Cambridge, MA: MIT Press, 1994

29 Oster G F, Wilson E O. Caste and Ecology in the Social Insects. Princeton, NJ: Princeton University Press, 1978

30 Shi L, Wang R W, Zhu L X, et al. Varying coefficient analysis for indeterminate species interactions with non-parametric estimation, exemplifying with a fig-fig wasp system. Chin Sci Bull, 2011, 56: 2545-2552

31 Frank S A. Policing and group cohesion when resources vary. Anim Behav, 1996, 52: 1163-1169

32 Ratnieks F L W, Wenseleers T. Policing insect societies. Science, 2005, 307: 54-56

33 Ratnieks F L W, Visscher P K. Worker policing in honeybees. Nature, 1989, 342: 796-797

34 Oldroyd B P, Ratnieks F L W. Anarchistic honey bee workers evade worker policing by laying eggs that have low remove rates. Behav Ecol Sociobiol, 2000, 47: 268-273

35 Halling L A, Oldroyd B P, Wattanachaiyingcharoen W, et al. Worker policing in the bee Apis florae. Behav Ecol Sociobiol, 2001, 49: 509-513

36 D'Ettorre P, Heinze J, Ratnieks F L W. Worker policing by egg eating in the ponerine ant Pachycondyla inversa. Proc R Soc Lond B, 2004, 271: $1427-1434$
37 Wenseleers T, Ratnieks F L W. Comparative analysis of worker reproduction and policing in eusocial hymenoptera supports relatedness theory. Am Nat, 2006, 168: 163-179

38 Duchateau M J. Agonistic behaviour in colonies of the bumblebee Bombus terrestris. J Ethol, 1989, 7: 141-151

39 Liebig J, Monnin T, Turillazzi S. Direct assessment of queen quality and lack of worker suppression in a paper wasp. Proc R Soc B, 2005, 272: 1339-1344

40 Keeling C I, Slessor K N, Higo H A, et al. New components of the honey bee (Apis mellifera L.) queen retinue pheromone. Proc Natl Acad Sci USA, 2003, 100: 4486-4491

41 Beggs K T, Glendining K A, Marechal N M, et al. Queen pheromone modulates brain dopamine function in worker honey bees. Proc Natl Acad Sci USA, 2007, 104: 2460-2464

42 Hollén L I, Manser M B. Motivation before meaning: Motivational information encoded in meerkat alarm calls develops earlier than referential information. Am Nat, 2007, 169: 758-767

43 Hollén L I, Radford A N. The development of alarm call behaviour in mammals and birds. Anim Behav, 2009, 78: 791-800

44 Bronstein J L. The costs of mutualism. Am Zool, 2001, 41: 825-839

45 Boyd R, Richerson P J. The evolution of reciprocity in sizeable groups. J Theor Biol, 1988, 132: 337-356

46 Weesie J. Asymmetry and timing in the volunteer's dilemma. J Confl Resol, 1993, 37: 569-590

47 Weesie J, Franzen A. Cost sharing in a volunteer's dilemma. J Confl Resol, 1998, 42: 600-618

Open Access This article is distributed under the terms of the Creative Commons Attribution License which permits any use, distribution, and reproduction in any medium, provided the original author(s) and source are credited. 